\title{
A Finite Element Modeling Proposal for Elucidating the Tensile Strength Characteristics Typically Observed in Natural Plant Fibers*
}

\author{
Kohji SUZUKI** \\ ${ }^{* *}$ Chiba Institute of Technology, Department of Mechanical Science and Engineering, \\ 2-17-1 Tsudanuma, Narashino-shi, Chiba 275-0016, Japan \\ E-mail: kohji.suzuki@it-chiba.ac.jp
}

\begin{abstract}
The purpose of this study is to elucidate factors affecting the statistical variations typically observed in the tensile strength of natural plant fibers such as kenaf bast fibers (KBFs) which recently have been looked upon as promising reinforcing fillers of environmentally-preferable polymeric composite materials. A statistical finite element modeling framework by combining beam and solid elements was proposed with both the variable cross-sectional geometries and the meso-scopic internal organic structures stochastically considered. The present modeling strategy was then effectively applied to the stress analysis and strength simulations of natural plant fibers under axial tension. From numerical examples in the case of $\mathrm{KBFs}$, it was shown that the meso-scopic internal organic structures, which consist of elementary fibrous cells (EFC) and inter-cell material (ICM), gave rise to axial stress concentrations in EFC and free-edge shear stresses in ICM, both of which should initiate the fiber fracture. Furthermore, by applying two-parameter Weibull analysis to the simulation data, the dependency of fiber strength and its initiating failure modes upon gauge length was also examined, which shows fairly good similarity with the actually-observed experimental results of monofilament tensile strength of natural plant fibers.
\end{abstract}

Key words: Natural Plant Fibers, FEM, Stress Analysis, Tensile Strength, Statistical Characterization, Weibull Distribution, Gauge Length Effect

\section{Introduction}

Natural plant fibers such as those extracted from bast layers within the stems of kenaf (Hibiscus cannabinus L.) will be able to clear the minimum requisitions as reinforcing fillers for polymeric composite materials while keeping plenty of their value-added environmentally-preferable advantages such as moderately high specific strength and stiffness, annually renewable with least $\mathrm{CO}_{2}$ discharge, affordable, abounding-everywhere, harmless-to-human and biodegradable features. Hence in recent years they have begun to be looked upon not only as raw materials in paper and textile industries but also as promising reinforcing fillers for both thermosetting and thermoplastic resins including biodegradable newcomers such as poly(lactic acid), PLA, in, for instance, automotive interior parts or consumer durable assemblies with strength, stiffness, heat resistance and short- to medium-term hygrothermal durability modestly required ${ }^{(1),(2)}$.

However, when we consider pushing this kind of plant biomass into the mainstream in the polymeric composites industries, we will probably hesitate, at least at this moment, to completely replace the conventional fibers, e.g. glass fibers (GFs), with those of natural 
plant origins such as kenaf bast fibers (KBFs). Since KBFs as well as any other natural plant fibers such as ramie, flax, hemp, sisal, bamboo fibers and so forth are not rigorouslyquality-managed industrial materials but rather merely agronomical crops, and have never been used in any durable composite structures before, the conventional engineering ways of testing and treating may not always be applicable to them, which certainly makes us feel insecure at the moment of taking them on the palm of our hands. For example, in the existing testing standards for composites reinforcing fibers such as glass, graphite and aramid, these conventional types of fibers are supposed to have perfectly uniform circular cross sections and the flaw distribution state which determines the fiber breaking stress range is also to be uniform not only within each fiber but among the population of fibers too. Unfortunately enough, these presuppositions will never apply to any natural plant fibers. In addition to this difficulty in testing, even the most carefully tested data for their mechanical properties, e.g. monofilament tensile strength, will still exhibit large scatters $^{(3),(4)}$. Due to the fact that natural plant fibers can only be produced through the biogenic activities of higher plants followed by being harvested and processed with traditional agricultural machineries or human labors, it is quite probable that the fiber geometries, morphologies, material compositions and preexisting damage states can be influenced sensitively by a lot of factors such as weather, district, harvest timing and methods and so forth, all of which have scarcely experienced being a research interest in materials and process engineering ever before and, more importantly, apparently are considerably hard and costly to control in an industrial manner. As one of quite suggestive examples, Bos et al. ${ }^{(5)}$ reported that the strength of flax bast fibers manually prepared with great care had a higher mean value than that of mechanically decorticated fiber strength, but coefficient of variation $(\mathrm{CV})$ of the former was larger than that of the latter because in the latter case fiber damage state, i.e. severity of kink bands formation ${ }^{(5)}$, was rolled off to its saturated level not only within each fiber but among fiber population as well, which ironically means that, from view points of strength reliability and quality assurance, the mechanically-damaged weak fibers are preferable to the carefully-handled strong fibers. Although a similar situation can often happen to any newly developed materials, it is mainly due to their premature process control, and usually it will gradually diminish as the manufacturing process is stabilized and optimized. The problem in the case of natural plant fibers, however, is that there are only either ad hoc manners, such as artificially introducing damages and mixing different lots, e.g. different years and districts of harvest, together just like "blended coffee beans", or otherwise costly ones, such as selecting truly intact fibers one by one, available for reducing such large $C V_{\mathrm{s}}$ exhibited in their physical properties. After all, from a practical view point, we have no choice but to take a standpoint that from the very beginning the geometrical and mechanical properties of natural plant fibers are statistically contaminated with scatter of little hope for improvement, and neglecting or failing in capturing such statistical variations might lead to a sort of unfavorable rumors that any test data for natural plant fibers is quite dubious and hence a margin of safety for their composite products should be set as much, but most likely unnecessarily, large as possible. Needless to say, that excessive margin of safety will result in the products being heavier, less functional, and, consequently, more environmentally-unfriendly.

One and only remedy for this problem will be after all to take a drastic reconstruction of the testing protocol properly taking into account the unique situation that the natural plant fibers are in while especially emphasizing their statistical characterization. For this final goal, there are so many things to do. First of all, accumulation of carefully-conducted test data and its expertise with emphasis on statistical aspects will be of utmost importance, to which the present author also has done not a few contributions in the case of kenaf bast fibers $(\mathrm{KBFs})^{(3),(4)}$. Similar, although not exactly the same, awareness of this issue also seems to be shared with a few recent researches concerning other natural plant fibers and 
composites as well as several over-forty-year longstanding activities around the paper and wood research fields ${ }^{(6),(7)}$.

Concurrently with testing, elucidation about what is going on inside the fibers at microand meso-scopic levels ought also to be addressed as a matter of priority ${ }^{(4)-(6)}$. Since there is a great deal of possibility that natural plant fibers, unless they are chemically regenerated like rayon and celluloid, retain their former appearance as a plant organ, their deformation, fracture and properties degradation behaviors as a composite reinforcing fiber can strongly reflect, and regrettably might be adversely affected by, their highly heterogeneous internal organic structures derived from such biological appearance in particular at meso-scopic level. Bos et al. ${ }^{(5)}$ investigated the tensile and compressive properties of both standard decorticated and hand isolated flax bast fibers for linen textile industry from the standpoint of their polymer composites application while relating them to kink bands formation and meso-scopic composite-like nature of this fibrous plant brand. According to their examinations on the tensile strength to cramping length (gauge length) relations for flax technical fibers, the tensile strength sharply decreased from $850 \mathrm{MPa}$ at $3 \mathrm{~mm}$ clamping length to the plateau value of $500 \mathrm{MPa}$ at or above $25 \mathrm{~mm}$. As an explanation for their observation, they set up a hypothesis that, since flax fibers themselves are a composite made up of elementary fibers, i.e. single plant cells, ranging $20-50 \mathrm{~mm}$ in length and pectin interface a few $\mu \mathrm{m}$ thick as gluing material, there would exist two clearly distinct kinds of failure mechanism, i.e. breakage of elementary fibers initiated from their preexisting kink bands for the clamping length below the elementary fiber length giving the flax fibers nearly maximum strength of $850 \mathrm{MPa}$ and shear failure (also they termed it splitting failure) in pectin interface for the clamping length larger than the elementary fiber length resulting in the fiber strength falling to 500MPa. They also presented three photos of a failed fiber tip in which both intact and halfway-broken elementary fibers could be seen entirely separated from each other, which they claimed partly supported their hypothesis. Although a possibility still remains that those elementary fiber separations could also have been induced by the elastic shock wave immediately after the fiber breakage, the present author are frequently finding similar kinds of separation in the case of kenaf bast fibers (KBFs) as well, which the author in turn call transverse cracks.

Bos et $a l^{(5)}$ also conducted general and environmental scanning electron microscope (SEM and ESEM) studies to seek further insights into the fracture behaviors in flax bast fibers. On the other hand, by using ESEM together with a digital image correlation (DIC) algorithm, Groom and his co-workers ${ }^{(6)}$ obtained a strain contour map of a black spruce fiber under stress, which showed that longitudinal strain concentrations in the proximity of pits on the surface were orders of magnitude greater than cell wall material that was defect-free or contained micro-compressions. It is no doubt that today's advanced ESEM technologies are extremely useful in elucidating the failure mechanisms of natural plant and wood fibers, but there is still a certain limitation for truly in-situ investigations mainly due to the presence of primary cell wall and lignin sediment covering over the fiber's outer surface $^{(5)}$. Although, other than ESEM, there are several experimental approaches available such as Raman spectroscopy ${ }^{(6)}$, confocal scanning laser microscopy ${ }^{(6)}$ and half fringe photoelasticity (HFP) for single filament composites $^{(6)}$, all of those experimental tools including ESEM can give us only limited and indirect information on the deformations and stress distributions in plant fibers and will need to be complemented by another totally different approach if one wants to firmly grasp the relation between the actual deformation and failure processes and the resulting phenomenological responses of the fibers.

In this context, the numerical analysis and simulation using finite element method (FEM) will emerge as one of the most powerful candidates for this purpose because such a numerical approach can reveal to us detailed deformation and stress distributions inside the natural plant fibers with randomly varying cross-sectional (CS) geometries and meso-scopic 
internal organic structures. In addition, the numerical approach can be used to virtually simulate the strength tests for any gauge length (GL) under any kinds of boundary conditions. However, to the present author's best knowledge, no one has taken any kinds of numerical approach for any natural plant fibers before mainly due to the difficulty to devise a modeling methodology for this analysis object with highly complex and stochastic nature.

In this study, for the purpose of elucidating factors affecting the statistical variations typically observed in the tensile strength of natural plant fibers, a statistical finite element modeling framework by combining beam and solid elements will be proposed with both the variable cross-sectional geometries and the meso-scopic internal organic structures stochastically considered after reviewing the experimental observations of the present author's own in the case of kenaf bast fibers (KBFs). Further, in accordance with the modeling methodology proposed in this study, a series of numerical examples by using two-dimensional finite element method (2-D FEM) will be carried out for the stress analysis and strength simulation of KBF monofilaments with various gauge length (GL) cases under axial tensile loading. Validity of the present 2-D FEM model will be discussed by comparing it to the experimental data, and then stress distributions in a single KBF specimen will be picked up and examined in detail to identify the locations and stress components that may initiate the fiber breakage. In addition, the strength simulation data will be subjected to the two-parameter Weibull analysis to see if the hypothesis of Bos et $a l{ }^{(5)}$ concerning the dependency of failure mechanisms upon GL is also a reality in the present KBF case.

\section{The Experimental Observations}

Experimental data acquisitions of the monofilament tensile strength of kenaf bast fibers (KBFs) at several different gauge length (GL) cases together with the thorough microscopic image analysis have already been conducted and reported by the present author and his co-workers. Those actual experimental observations will be briefly reviewed in this chapter. More detailed descriptions on these experiments can be referred to elsewhere ${ }^{(3),(4)}$.

\subsection{Experimental procedures}

Intact kenaf bast fibers (KBFs) were manually and randomly sampled with the possible care from an identical intermediate portion of an individual plant stalk (harvested by the present author $\left.{ }^{(8)}\right)$, and then glued onto a C-shaped kraft paperboard with jelly-like instant glue with gauge length (GL) varied from 2 up to $40 \mathrm{~mm}$. Load-elongation curves for each of the specimens until its breakage were obtained using an instron-type screw-driven universal tester at $0.5 \mathrm{~mm} / \mathrm{min}$ crosshead speed. Each of the after-the-test specimens was next buried in EVA(ethylene vinyl acetate copolymer) resin, and was transversely cut in round slices with a handy microtome along the whole GL at $200 \mu \mathrm{m}$ interval. These sequential sliced pieces were then subjected to the PC-based optical-microscopy image analysis to quantify the fiber cross-sectional (CS) geometries, such as CS area, as well as to clarify their biological internal structures.

\subsection{Monofilament tensile strength of KBFs}

An effective cross-sectional (CS) area, $\bar{A}$, for strength evaluation was determined by taking a sample mean (leveled-off-within-fiber) value over the set of the envelope areas actually measured from the continuously-sliced CS images, and then a strength, $\sigma_{a}$, of each specimen was evaluated by straightforwardly dividing the breaking load, $P$, by that effective CS area, $\bar{A}$, i.e., $\sigma_{a}=P / \bar{A}$. Figure 1 shows the histogram of the strength $\sigma_{a}$ variation for sample size, $n=66$. The sample mean and sample coefficient of variation $(C V)$ of $\sigma_{a}$ for all gauge length (GL) cases put together were found to be $309 \mathrm{MPa}$ and $43.3 \%$ respectively. It 


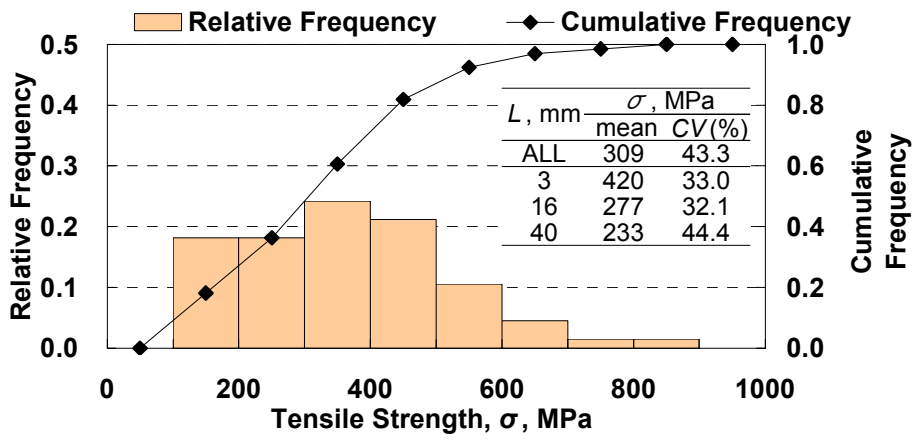

Fig.1 Histogram of monofilament tensile strength of KBFs

should be noted that the value of cross-sectional area at fracture point, which is difficult to measure after testing unfortunately, might be also a candidate as an effective value.

According to Weibull analysis, the distribution function, $F$, for the data of fiber strength, $\sigma$, is supposed to obey the two-parameter Weibull distribution when it is expressed as,

$$
F(\sigma ; L)=1-\exp \left[-\left(\frac{L}{L_{0}}\right) \cdot\left(\frac{\sigma}{\sigma_{0}}\right)^{m}\right]
$$

where $L$ is the gauge length (GL) of the specimen, and $m$ and $\sigma_{0}$ stand for the shape and the scale parameters respectively, and $L_{0}$ is the characteristic length. In Fig.2(a), Weibull probabilistic plots for tensile strength $\sigma_{a}$ of three different GLs, $L=3,16$ and $40 \mathrm{~mm}$, are

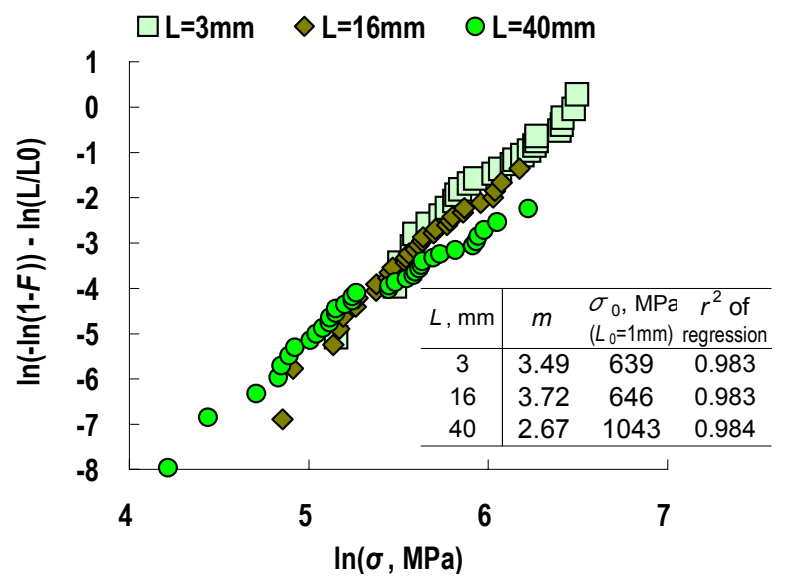

(a)

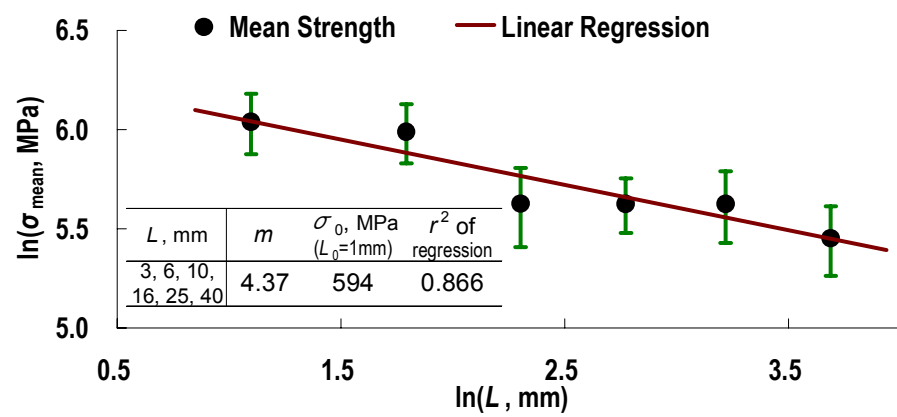

(b)

Fig.2 Two-parameter Weibull probabilistic plots and parameters estimated by linear regressions for the monofilament tensile strength of KBFs 
shown. Every case of those different GLs forms a nearly straight line (every determination coefficient, $r^{2}$, was well above 0.98), which implies that strength of kenaf bast fibers (KBFs) basically can be fit to a Weibull-type distribution based on the so-called weakest-link theory. In Fig.2(b), on the other hand, the mean strength, $\sigma_{\text {mean }}$, is plotted against GL in the double logarithmic scales. The error bar attached at each data show upper and lower $95 \%$ confidence limits for the population mean. Obviously the strength has a fairly strong negative correlation to GL, as is often seen in other brittle engineering fibers used for polymeric composites reinforcement such as E-glass and graphite fibers. However, the bi-linear relation reported by Bos et al. ${ }^{(5)}$ for flax fiber strength was not seen in this GL range for KBF strength probably because the elementary fiber (elementary fibrous cell (EFC)) length of KBFs $(2-3 \mathrm{~mm})$ is much smaller than that of flax fibers $(20-50 \mathrm{~mm})$. The shape parameters, $m$, estimated from the plots in Fig.2(a) for the GLs, $L=3$, 16, and 40mm were respectively $3.49,3.72$, and 2.67 , while the one estimated from the regression line in Fig.2(b) turned out to be 4.37 , by $17.5-63.7 \%$ greater than those from the plots in Fig.2(a). The discrepancy between these parameter estimations for the same strength data is apparently alerting that the classical two-parameter Weibull distribution model may be practically applicable only to the strength data with GL fixed but not completely appropriate for describing the mono-filament tensile strength of KBFs with GL varied.

\subsection{Variable CS geometries of KBFs}

Two kinds of optical micrographs shown in Fig.3 are respectively mutuallyperpendicular side-views (the upper) and sequentially sliced cross-sectional (CS) images (the lower) of single kenaf bast fibers (KBFs), both of which were typically observed in the experiment. From these micrographs, the following facts regarding the KBF geometry can be depicted; (a) the actual cross sections of the fibers are not a circular but an ellipse-like irregular polygon having a number of inside open holes (lumens), and (b) the fibers themselves may be twisted around even though any torsional loading is not applied.

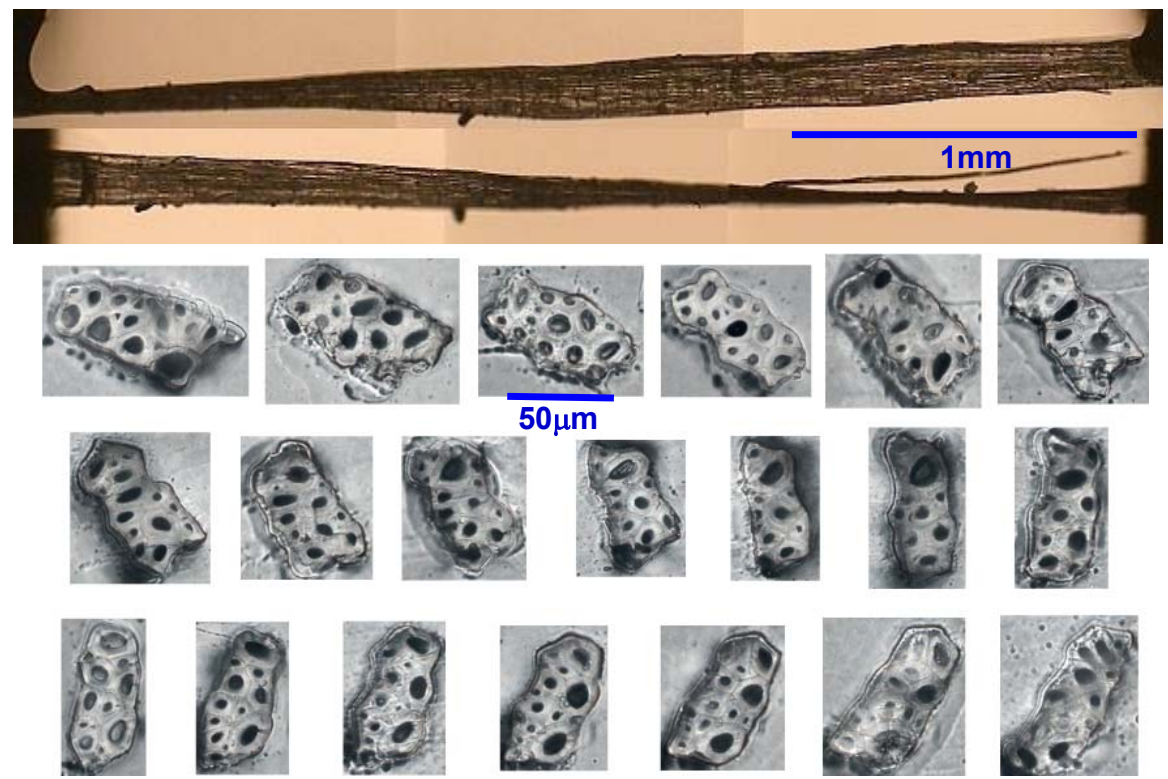

Fig.3 Mutually-perpendicular side-views and sequentially sliced CS images of single KBFs

Figure 4 is one of the typical along-fiber profiles for the CS area measurements. Considerable variation of CS area within a fiber can be recognized from that profile. For the purpose of quantifying such CS area variation along a single fiber, leveled-off-within-fiber $\mathrm{CS}$ area, $\bar{A}$, and within-fiber coefficient of variation, $C V_{A}$ may be the most useful. In this 
case, $\bar{A}$ and $C V_{A}$ were found to be $5695 \mu \mathrm{m}^{2}$ and $18.1 \%$ respectively.

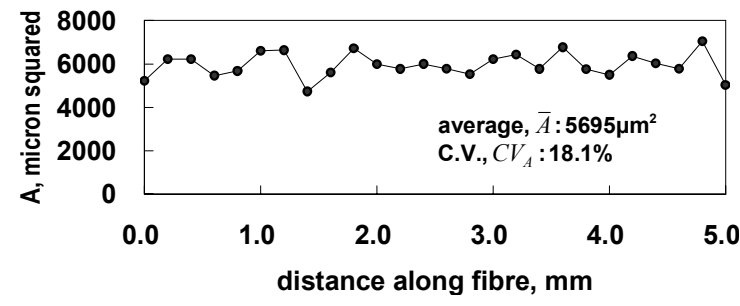

Fig.4 A typical case of along-fiber variations of KBF CS area

Kenaf bast fibers (KBFs) exhibit geometrical variations not only within each single fiber but among a collection of fibers as well. Figures 5(a) and (b) respectively show sample histograms of the leveled-off-within-fiber cross-sectional (CS) area, $\bar{A}$, and the withinfiber coefficient of variation, $C V_{A}$. Both $\bar{A}$ and $C V_{A}$ vary widely even if the sample were taken from an identical organ in an individual plant. Note also that it was confirmed by the present author that both $\bar{A}$ and $C V_{A}$ could be fit well to the normal distributions and that there was no correlation between them at all. Possibly along- and among-fiber variations in terms of fiber CS area (or diameter) will affect fiber strength. Zhang et al. ${ }^{(9)}$ modified the Weibull/weakest-link model so as to incorporate the within-fiber diameter variation for wool fibers, and verified its accuracy and consistency for wool fiber strength of any gauge length (GL) range. Although their work should be further extended to incorporate among-fiber variation as well as along-fiber, it is certainly one of worthwhile contributions to pose the importance of considering CS geometry variations in strength distributions of natural fibers.

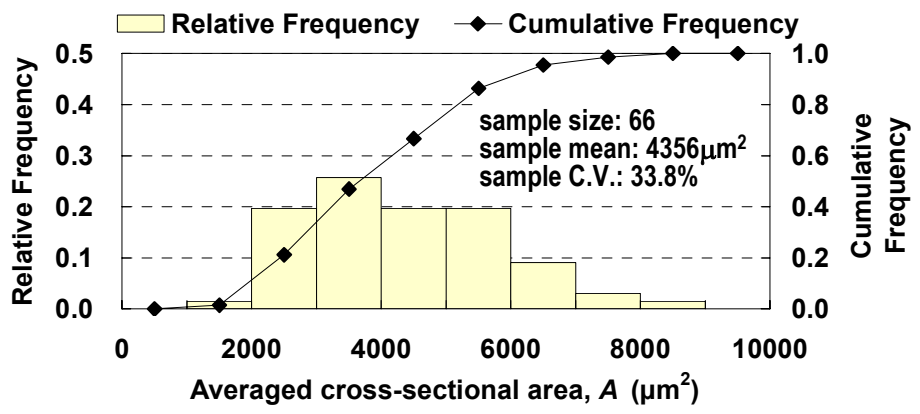

(a)

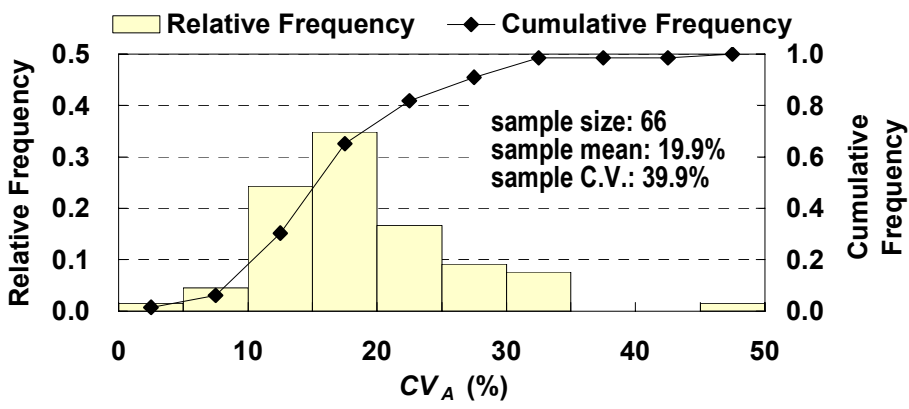

(b)

Fig.5 Among-fibers variations of KBF CS area related quantities 


\subsection{Meso-scopic internal structure of KBFs}

Figure 6 illustrates the typical internal structures of a kenaf bast fiber (KBF). In the meso-scopic level, KBFs, as can also be seen in any other natural plant fibers such as flax bast fibers ${ }^{(5)}$, are definitely not a monolithic and homogeneous single filament having a uniform circular cross section but rather a bundle or a composite consisting of 10-30 spindle-shaped plant cells, which, in the KBF case, are approximately $17-21 \mu \mathrm{m}$ wide and 2.4-3.3mm long ${ }^{(10),(11)}$ and randomly packed into the polygonal section. The cells are mostly constructed of the cellulose-rich secondary cell wall. In this study, those plant cells, also termed ultimate fibers ${ }^{(10)}$ or elementary fibers ${ }^{(5)}$, are to be called "elementary fibrous cell (EFC)". One of the most prominent features commonly seen in EFC morphology is an inside open hole, which is called "lumen". In addition to EFC, "inter-cell material (ICM)", which has also been called middle lamella ${ }^{(12)}$ or pectin interface ${ }^{(5)}$, is also an important constituent for mechanical properties of natural plant fibers. It is a kind of binder a few $\mu \mathrm{m}$ thick gluing the neighboring EFCs together. This adhesive material is primarily made up of non-cellulose polysaccharides such as pectin, hemicellulose, and, especially in case of mature fibers, lignin ${ }^{(12),(13)}$. Although its stiffness and strength are considerably small when compared to those of EFC, almost all of the loads from one EFC to the others will be carried via the shear stresses in this interfacial region, and hence there is a possibility that the mechanical properties of ICM govern the overall mechanical performance of KBFs.

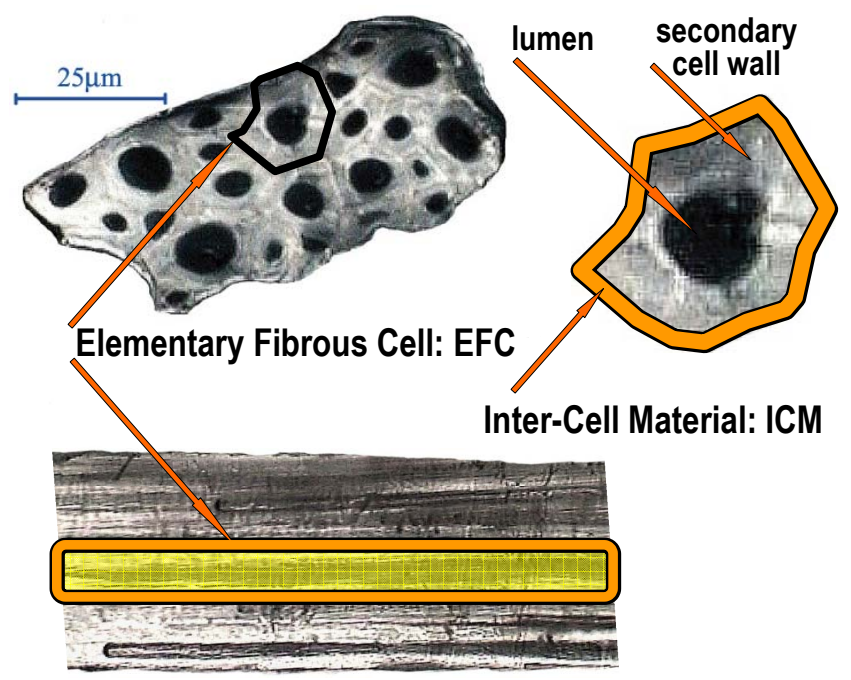

Fig.6 Meso-scopic internal structures of KBF

The variable cross-sectional (CS) geometries and meso-scopic internal structures made up of EFCs and ICM observed by the present author in the KBFs case and briefly reviewed in this chapter are very specific to natural plant fibers and will never exist in their synthetic counterparts, e.g. E-glass fibers. In order to firmly grasp the statistical nature of KBF strength and then to construct a plausible distribution that can be successfully served in their material and composite tests, designs, processing and strength reliability and quality assurance for the products, it will be crucial to investigate whether or not and, if it is so, how much those features of KBFs affect their global strength responses against various kinds of external loading and conditions. In the following chapter, a numerical approach based on finite element method (FEM) will be employed for answering, even if only partially, those questions. 


\section{Finite Element Modeling Proposal}

\subsection{FEM modeling framework; a case study for KBFs}

Finite element (FE) modeling of natural plant fibers is a computationally challenging topic since they are inherently natural products with randomly varying geometries and highly heterogeneous internal organic structures. Variation or uncertainty in their material properties must also be large as compared to that of process-controlled materials. These facts on this analysis object may make us abandon fully three-dimensional (3-D) modeling by using continuum solid FEs. In this study, instead of a fully 3-D continuum solid FE modeling, the following simplified modeling framework by a combination of beam and continuum solid finite elements is proposed for the stress analysis and strength simulation of natural plant fibers such as kenaf bast fibers (KBFs). As schematically shown in Fig.7, elementary fibrous cells (EFCs) are to be meshed with beam FEs. The cross-sectional (CS) figurations, such as CS area, $A_{\mathrm{EFC}}$, height, $h_{\mathrm{EFC}}$, second moment of CS area and so forth, of EFCs can vary along their length, so they will need to be probabilistically sampled from an appropriate distribution together with its mean and coefficient of variation $(C V)$. Similarly, the length of EFC, $L_{\mathrm{EFC}}$, and its axial strength, $X_{\mathrm{EFC}}$, should also be allowed to vary. Spatial arrangement of EFCs within each fiber were iteratively created by invoking the uniform random distribution. On the other hand, inter-cell material (ICM) regions are to be meshed with continuum solid FEs having the same nodes in common with the EFC beam FEs. All the geometrical and material properties assigned to EFC and ICM FEs were determined after discussed with the present author's best effort referring to some existing, but quite limited, literatures ${ }^{(10)-(16)}$ and the in-house experimental data of the present author's own.

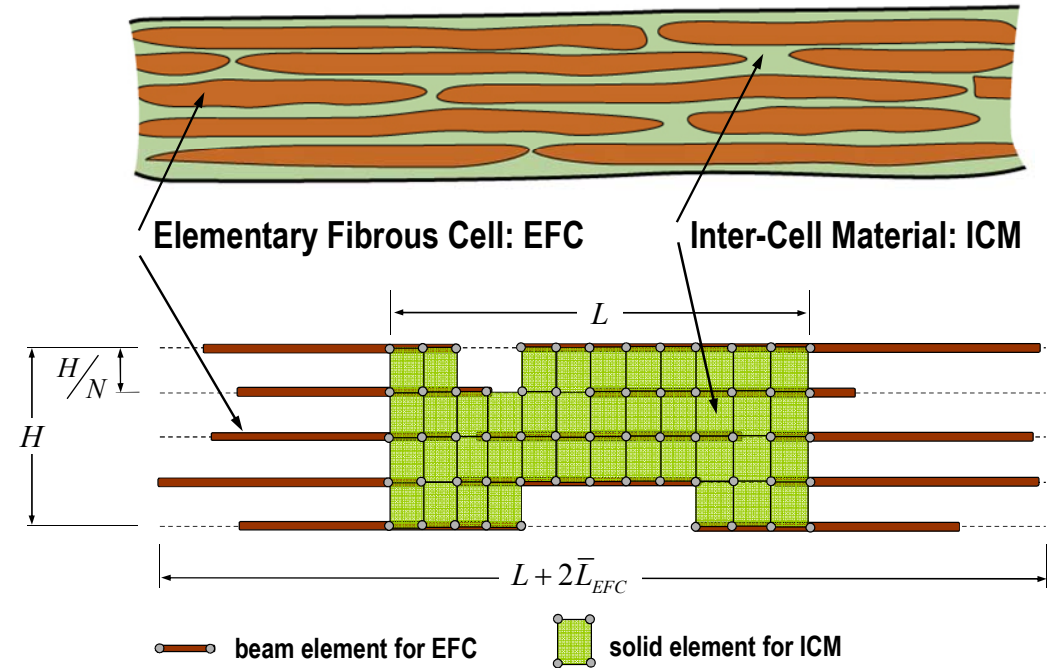

Fig.7 Modeling methodology of KBFs by beam and solid FEs

The FE modeling specifications that the present author actually assumed for KBFs are as follows. First the fiber section height, $H$, and the maximum number of EFCs across the section, $N$, were defined and respectively assumed to be $100 \mu \mathrm{m}$ and 11 . For the sake of simplicity, the fiber section height, $H$, was kept constant at the above-mentioned value and EFCs were evenly arranged across the section. The fiber whole length was defined as $L+2 \bar{L}_{\mathrm{EFC}}$, in which $L$ is the gauge length (GL) of the specimens, which was varied from 0.1 to $40 \mathrm{~mm}$ in the strength simulation, and $\bar{L}_{\mathrm{EFC}}$ is the mean of EFC length, $L_{\mathrm{EFC}}$, which was assumed $3 \mathrm{~mm}$ in this study. $L_{\mathrm{EFC}}$ was assumed to vary according to the normal distribution with the above mean value and the standard deviation $(S D)$ of $0.6 \mathrm{~mm}$ (i.e., $C V=20 \%$ ). $\mathrm{EFC}$ 
arrangement within the fiber was started from the center. The position in the along-fiber direction for each EFC was iteratively determined by generating uniform pseudo random numbers. If a certain position for an EFC had already occupied by other precedent, then another random number was generated for the next trial. This iteration for EFC arrangement in an along-fiber direction was terminated at 200 times and then that arrangement routine was shifted to one outer location in the across-fiber direction. This iteration continued until the outermost arrangement was terminated. The reason why the fiber whole length for EFC to be arranged is by twice of the EFC mean length longer than the GL for strength evaluation is to avoid the EFC arrangement configurations at or around the GL ends being different from those on the inside. After that, CS area, $A_{\mathrm{EFC}}$, with $100 \mu \mathrm{m}^{2}$ mean and $50 \mu \mathrm{m}^{2}$ $\mathrm{SD}(50 \% C V)$, height, $h_{\mathrm{EFC}}$, with $10 \mu \mathrm{m}$ fixed and Young's modulus, $E_{\mathrm{EFC}}$, with $70 \mathrm{GPa}$ fixed were assigned to each EFC. FE model employed in this study didn't consider the scatter in angles of EFCs. Its effect on tensile behavior might be smaller than that on compressive behavior. In case of twisting and bending problems, such features of EFCs should be included. Only those EFCs in the inner ward GL region of the fiber were then discretized with 2-node beam FEs of $100 \mu \mathrm{m}$ length. To the generated EFC beam FEs, axial strength, $X_{\mathrm{EFC}}$, with $1 \mathrm{GPa}$ mean and $100 \mathrm{MPa} S D(10 \% \mathrm{CV})$ were assigned. All the probabilistic quantities were assumed to be in the normal distributions. On the other hand, ICM was modeled by filling the gaps between adjacent EFC beam FEs with 2D plane-stress 4-node solid FEs. If the distance between two adjacent beam FEs was greater than twice of the minimum distance, i.e. $H / N$, then ICM solid FEs were not created to such regions. Young's modulus, $E_{\mathrm{ICM}}$, and Poisson's ratio, $v_{\mathrm{ICM}}$, assigned to ICM solid FEs were assumed 10GPa and 0.3 respectively. Finally, to the generated ICM solid FEs, shear strength, $S_{\mathrm{ICM}}$, of a normal distribution with $60 \mathrm{MPa}$ mean and $12 \mathrm{MPa} S D(5 \% \mathrm{CV})$ were assigned. As previously mentioned, the major ingredients of this cementing material are polysaccharide fractions such as pectin and hemicellulose. There is no observation on existence of cellulose contents in this interfacial regions, and therefore its stiffness and strength are considerably small as compared to those of EFC that is known to be reinforced with cellulose.

\subsection{Monofilament tensile strength simulation for KBFs}

For the purpose of examining how the fiber strength depends on gauge length (GL), thirteen GL cases, $L=0.1,0.3,0.5,1,2,3,4,5,6,10,16,25$ and $40 \mathrm{~mm}$, were simulated in this study. The size of sample (the number of specimens), $n$, was initially set to 50 for every GL case, but, particularly for the shorter GL cases, the actual sample size tended to become smaller than that initially-set size because the random arrangement of EFCs carried out in this study, particularly in very short fiber length ranges, had not always succeeded when the iterations for arrangement were terminated at 200 times.

At one GL end of each specimen was fixed and forced displacement, $\Delta L$, was longitudinally applied at the other GL end. Fiber cross-sectional (CS) area was determined in the same manner as those in the author's experimental study, which has already explained earlier in this paper, that is, an effective CS area, $\bar{A}$, of each specimen was calculated as a mean (leveled-off-within-fiber) value for the actual CS areas across the fiber. The individual across-fiber CS areas at each FE discretization unit were so obtained as a sum of those of EFC beam FEs at that unit. Monofilament tensile strength, $\sigma_{a}$, of each FE specimen was evaluated from an axial reaction force, $P$, for the first failure of the FE model and the effective CS area, $\bar{A}$, i.e., $\sigma_{a}=P / \bar{A}$. The failure load, $P$, was judged at the first moment when the stress in a certain element exceeds its strength. The components of stress and strength for that comparison were the axial tensile component for EFC beam FEs and the shear component for ICM solid FEs. This fracture judgment will be sufficiently applicable to brittle materials and the load-elongation curves of KBFs obtained by the present author certainly indicates the nature of brittle fracture ${ }^{(3),(4)}$. This kind of brittle nature is also 
reported by Bos et al. ${ }^{(5)}$ in the case of flax bast fibers. In general, for ordinal plant fibers, tracking their progressive failure process will not be necessary since the overall fiber breakage seems to instantaneously occur immediately after the internal fracture on-sets.

\section{Numerical Examples and Discussion}

\subsection{Stress distribution in a KBF FE model}

In Fig.8, a typical FE meshes (the first specimen for the simulation of gauge length (GL), $L=5 \mathrm{~mm}$ ) are shown.

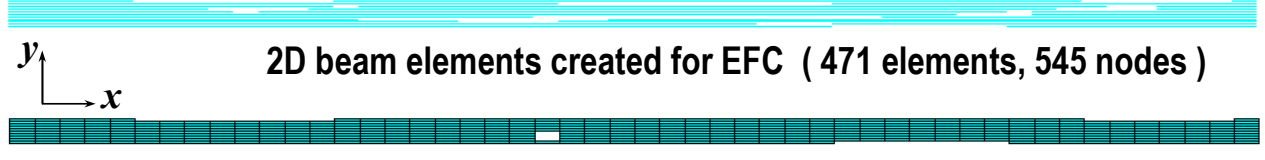

2D plane strain solid elements created for ICM ( 475 elements )

Fig.8 A FE mesh created for the first specimen of 5mm-GL KBFs

Figure 9 is the along-fiber cross-sectional (CS) area profile of the same specimen as shown in Fig.8, which clearly shows that the CS area varies along the FE model accompanying with localized variation of EFC sparseness. In this case, leveled-off-withinfiber $\mathrm{CS}$ area, $\bar{A}$, and within-fiber coefficient of variation, $C V_{A}$ were respectively $942 \mu \mathrm{m}^{2}$ and $10.1 \%$. By the way, these values in terms of geometrical quantity generated in the present simulation can not directly be compared to the experimental measurements such as those shown in Fig.4, since the present FE model is 2-D with several assumptions and simplifications for the specimen geometries as well as the material properties. Nonetheless the present author believe that the present model may be a reasonable first attempt for extracting the essence and stepping further into the reality.

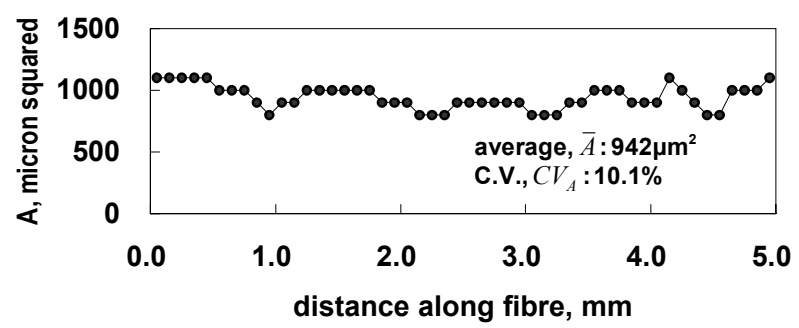

Fig.9 Cross-sectional area variation along the FE specimen

In Figs.10(a) through (c), respectively, distributions of longitudinal normal stress (i.e., axial stress), $\sigma_{x}$, in EFC beam FEs, and transverse shear stress, $\tau_{x y}$, and Mises equivalent stress in ICM solid FEs for the same 5mm-GL specimen as in Figs. 8 and 9 under $\Delta L / L=$ $5000 \mu$ loading are shown. For easiness to see, these contour plots are by five times enlarged only in the vertical direction. Owing to the KBF meso-scopic internal structure, stress states in the fiber are definitely not uniform but rather like those often seen in unidirectional short-fiber or partly-broken-fiber reinforced composites. As seen in Fig.10(a), there are a few local regions of high EFC axial stresses clearly observed around the mid-spans of, say, isolated EFCs that are missing neighboring EFCs. It is readily understood that overall fiber breakage can be initiated by one or a few local EFC breakages caused by those localized 
high axial stresses and such high axial stress regions can naturally occur in sections of smaller fiber CS area. In Fig.10(b), on the other hand, several point-wise concentrations of ICM transverse shear stresses in the vicinities of where EFCs suddenly terminate were also recognized. Theoretically those concentrations, or singularities, of the ICM shear stresses at the specific sites where the axial stress in EFC suddenly disappear can be explained by self-equilibrium of internal forces, and is well known as the free-edge effects in the elasticity of laminated composites ${ }^{(17)}$. Although it will depend on 3-D geometries of the EFC ends and plastic nature of ICM, those locally concentrated shear stresses (i.e., free-edge stresses) in ICM can also be a starting point of overall fiber breakage, while competing or affecting to each other with the axial stresses in EFC. By the way, speaking of plasticity of ICM, high Mises equivalent stresses as seen in Fig.10(c) that will form plastic yield regions there in ICM are found to wrap both the high EFC axial stresses and the singular ICM shear stresses, which implies that ICM plasticity might also play more or less an important role in the actual KFB fracture process especially when one wants to quantitatively compare the numerical results to the experiments, as is often necessary in the case of thermoplastic tough resin composites, although any further discussions on this topic will be left to a future study. In addition, one of the greatest differences of the present 2-D FEM model from the actual 3-D KBFs may be in that it does not have any modeling capability for the torsional mode (i.e., mode III as termed in the fracture mechanics), and, recalling that KBFs are twisted around as shown in Fig.3, this may also be another important topic that should be put with priority on a future to-do-list.

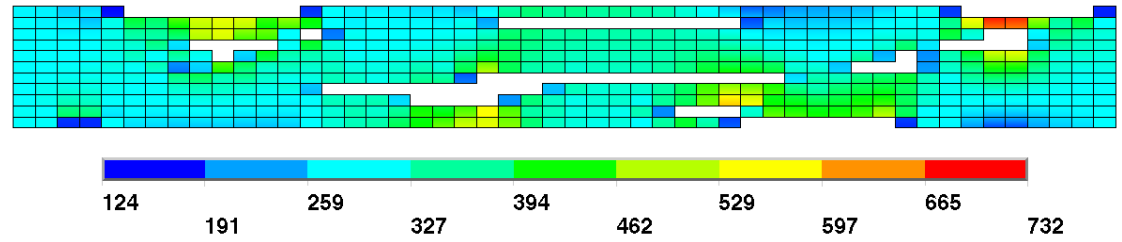

(a)

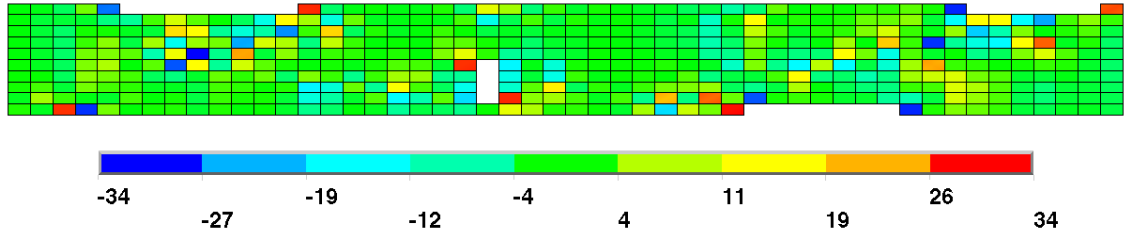

(b)

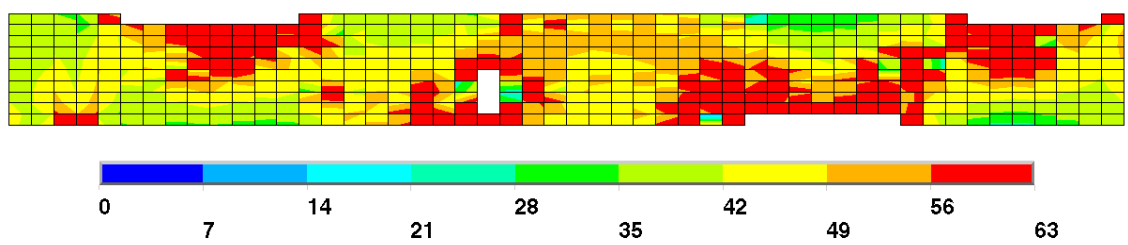

(c)

Fig.10 Stress distributions for (a) $\sigma_{x}$ in EFC, and (b) $\tau_{x y}$ and (c) Mises equivalent stress in ICM at $5000 \mu \varepsilon$ tensile loading (in MPa)

\subsection{Statistical strength simulation by using 2-D FEM}

Figures 11(a) and (b) respectively show histograms of leveled-off-within-fiber crosssectional (CS) area, $\bar{A}$, and within-fiber coefficient of variation, $C V_{A}$ for the $5 \mathrm{~mm}-\mathrm{GL}$ sample consisting of 50 specimens. Since the fiber section height, $H$, for EFC arrangement was kept constant at $100 \mu \mathrm{m}$ along the fiber, variation of $\bar{A}$ among fibers was not so large 
as that in the experiment shown in Fig.5(a). In addition, the sample mean of $\bar{A}$ was nearly one fifth of the experimental result. This is, as mentioned earlier, because total number of EFCs in the present 2-D FEM models were considerably smaller than that of the actual fibers. However, it will be interesting to note that the among-fiber variation of $C V_{A}$ in the simulation was found to be fairly comparable to the experimental in Fig.5(b).

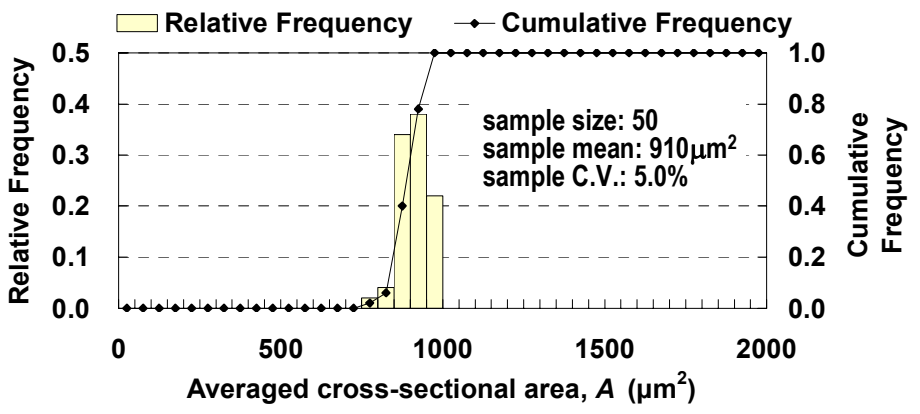

(a)

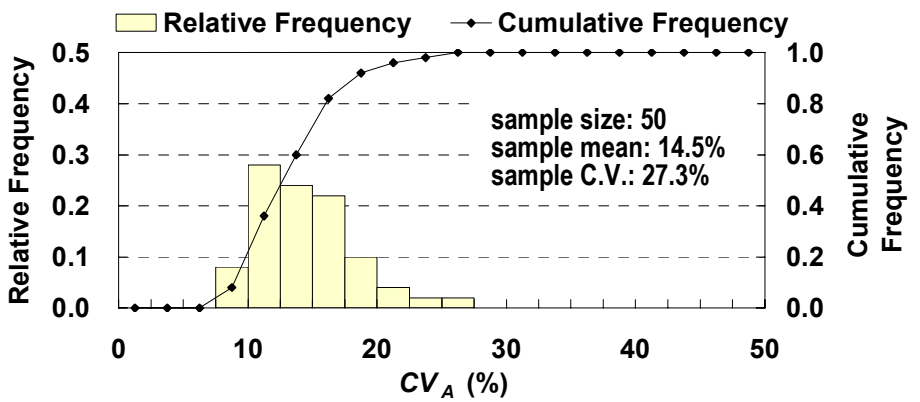

(b)

Fig.11 Histogram of the cross-sectional area among the FE models created during the strength simulation

In Fig.12, the histogram of the strength $\sigma_{a}$ for the same $5 \mathrm{~mm}-\mathrm{GL}$ sample is shown. The sample mean and sample coefficient of variation $(\mathrm{CV})$ of $\sigma_{a}$ for this GL case were $319 \mathrm{MPa}$ and $23.0 \%$ respectively.

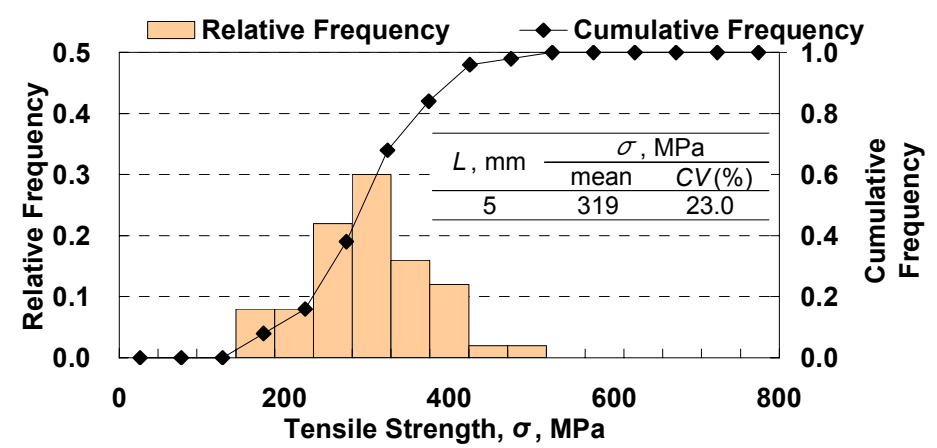

Fig. 12 Histogram of the monofilament tensile strength for the FE models created during the strength simulation

As in the similar fashion to the experimental strength data analysis, the strength $\sigma_{a}$ data for all of the thirteen different GL simulations are simultaneously plotted on a single 
Weibull probabilistic chart in Fig.13(a) together with the estimated Weibull parameters from linear regression to the plots only for the eight selected GL cases. Every case of the simulation results was found to fit practically well to the two-parameter Weibull distribution. However the shape and scale parameters respectively estimated from the different GLs most likely differed from each other; Seemingly the shape parameters, $m$, estimated from shorter GL data are apt to become greater than those estimated from the longer GL data. On the other hand, Figure 13(b) is a double logarithmic plot for the mean strength, $\sigma_{\text {mean }}$, against the GL. The shape parameter, $m$, estimated from this plot turned out to be 4.04, which was larger than those estimated from the single Weibull plots for the longer GL data in Fig.13(a) and smaller than those for the shorter. It is well-known that the shape parameter, $m$, of two-parameter Weibull distribution can be described with the coefficient of variation, $C V$, only, as,

$$
C V=\left\{\frac{\Gamma(1+2 / m)}{\Gamma^{2}(1+1 / m)}-1\right\}^{\frac{1}{2}}
$$

in which $\Gamma$ is gamma function and the greater $C V$ simply corresponds to the smaller $m$. Therefore, one of the desirable directions toward understanding these simulation results

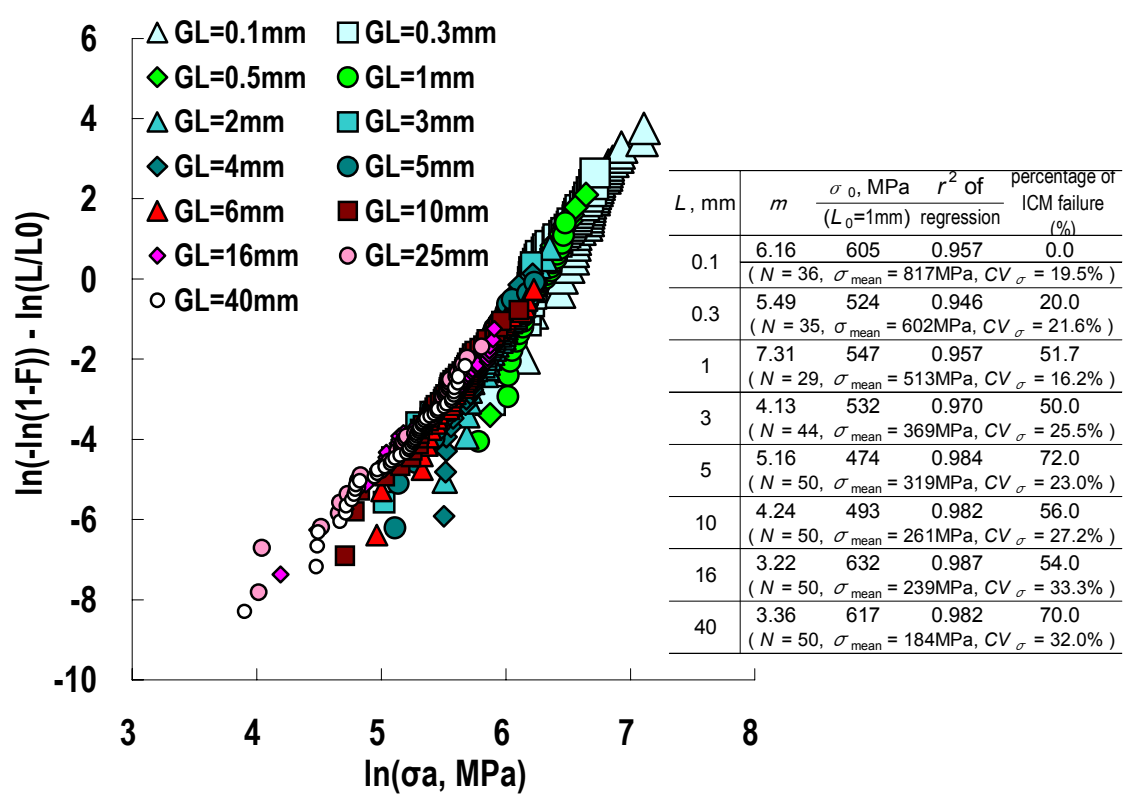

(a)

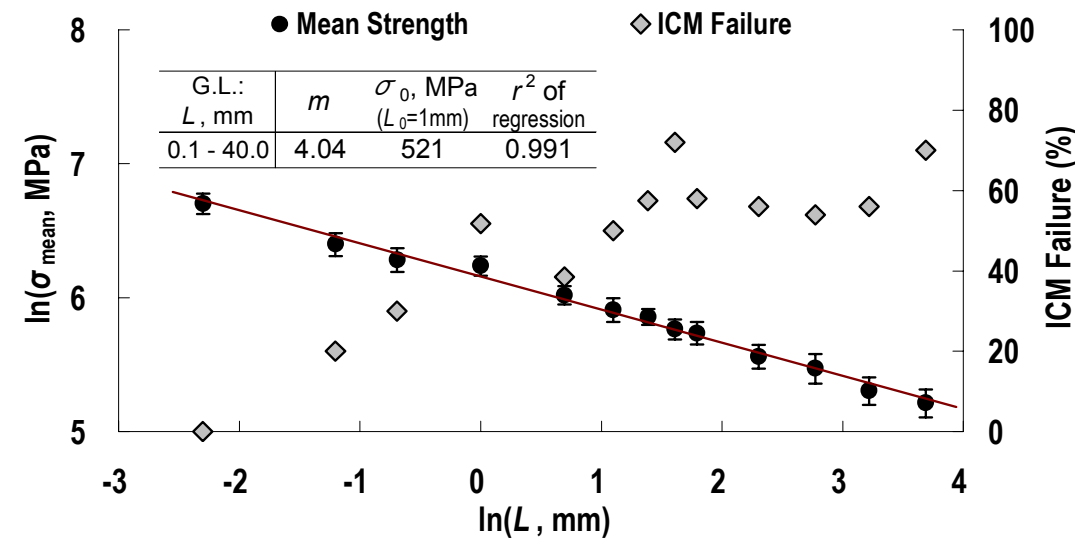

(b) 
might be to investigate the factors affecting $C V$ (or, more generally, variation) of the simulation data with it related to GL.

One of such factor affecting the $C V$ behaviors with regard to GL will be EFC sparseness variation along the fiber. It has already been known that EFC has a certain peculiar (mean) length, which was assumed $3 \mathrm{~mm}$ in the present simulation, and when specimen GL is shorter than the EFC length, probability of sparse EFC arrangements will become very small as compared to the longer-than-EFC GL cases. Strength for a specimen without any EFC sparseness variation will be exclusively determined by axial strength of EFCs since stress distributions in such a specimen will be quite monotonous merely depending upon the CS area of each EFC and having less probability for ICM shear stress singularity. This will be one of the reasons why $C V \mathrm{~s}$ of strength (i.e., shape parameter, $m$ ) for very short GLs are relatively small (i.e., for $m$, large). When the specimen GL is increased, the probability of EFC sparseness arrangements will become larger, and at or beyond a certain length, that probability will reach, say, its saturated value. In such a longer GL specimen, its strength will be determined by either EFC axial tensile breakage or ICM shear failure. In Fig.13(a) and (b), percentage of specimens which were seen broken (in the sense of first failure) from the ICM shear failure mode. The percentage in Fig.13(b) is the one for each GL, in which an ICM element fractured in advance of the fracture of EFC element. As can be seen, percentage of the ICM shear failure specimens increases as GL increases in the range from $0.1 \mathrm{~mm}$ to $1 \mathrm{~mm}$ and for GLs of longer than $1 \mathrm{~mm}$, that percentage, although still fluctuating, seems constant at approximately $56 \%$ on average. These results numerically explain the hypothesis by Bos et al. ${ }^{(5)}$ referred in the introduction chapter.

\section{Conclusions}

In this study, a statistical finite element modeling framework by combining beam and solid elements was proposed with both the variable cross-sectional geometries and the meso-scopic internal structures stochastically considered. The present modeling strategy was then effectively applied to the stress analysis and strength simulations of natural plant fibers under axial tension. From numerical examples in the case of kenaf bast fibers (KBFs), it was found that the meso-scopic internal structures in KBFs, which consist of elementary fibrous cells (EFC) and inter-cell material (ICM), gave rise to axial stress concentrations in EFC and free-edge shear stresses in ICM, both of which should initiate the fiber fracture. Furthermore, by applying two-parameter Weibull analysis to the simulation data, the dependency of fiber strength and its initiating failure modes upon gauge length was also investigated, which shows fairly good similarity with the actually-observed experimental results of monofilament tensile strength of natural plant fibers.

\section{References}

(1) Serizawa, S., Inoue, K., and Iji, M., Kenaf-Fiber-Reinforced Poly(lactic acid) Used for Electric Products, Journal of Applied Polymer Science, Vol.100, No.1 (2006), pp.618-624.

(2) Lee, B.-H.,, Kim, H.-S., Lee, S., Kim, H.-J., and Dorgan, J.R., Bio-Composites of Kenaf Fibers in Polylactide: Role of Improved Interfacial Adhesion in the Carding Process, Composites Science and Technology, Vol.69, No.15-16 (2009), pp.2573-2579.

(3) Suzuki, K., Kimpara, I., and Funami, K., On Tensile Strength Properties of Natural Bast Fibers Taken from Dicotyledonous Plants, Proceedings of $2^{\text {nd }}$ International Workshop on "Green Composites" (IWGC-2), Ube-shi, (2004), pp.59-63.

(4) Suzuki, K., Kimpara, I., Saitoh, H., and Funami, K., Cross-Sectional Area Measurement and Monofilament Strength Test of Kenaf Bast Fibers (in Japanese), Journal of the Society of 
Materials Science, Japan, Vol.54, No.8 (2005), pp.887-894.

(5) Bos, H.L., Van Den Oever, M.J.A., and Peters, O.C.J.J., Tensile and Compressive Properties of Flax Fibres for Natural Fibre Reinforced Composites, Journal of Materials Science, Vol.37, No.8 (2002), pp.1683-1692.

(6) Eichhorn, S.J., Baillie, C.A., Zafeiropoulos, N., Mwaikambo, L.Y., Ansell, M.P., Dufresne, A., Entwistle, K.M., Herrera-Franco, P.J., Escamilla, G.C., Groom, L., Hughes, M., Hill, C., Rials, T.G., and Wild, P.M., Current International Research into Cellulosic Fibres and Composites, Journal of Materials Science, Vol.36, No.9 (2001), pp.2107-2131.

(7) Nechwatal, A., Mieck, K.-P., and Reußmann, T., Developments in the Characterization of Natural Fibre Properties and in the Use of Natural Fibres for Composites, Composites Science and Technology, Vol.63, No.9 (2003), pp.1273-1279.

(8) Suzuki, K., Research Report on Test Growing and Fiber Extraction of Kenaf, Report of Chiba Institute of Technology, No.51 (2004), pp.145-152.

(9) Zhang, Y., Wang, X., Pan, N., and Postle, R, Weibull Analysis of the Tensile Behavior of Fibers with Geometrical Irregularities, Journal of Materials Science, Vol.37, No.7 (2002), pp.1401-1406.

(10) Khristova, P., Bentcheva, S., and Karar, I., Soda-Aq Pulp Blends from Kenaf and Sunflower Stalks, Bioresource Technology, Vol.66, No.2 (1998), pp.99-103.

(11) Shah, S.M.A., Younis, M.T., and Nabi, G., Characteristics of Kenaf (Hibiscus Cannabinus) Fibers. Part I. Physical Characteristics, Pakistan Journal of Scientific and Industrial Research, Vol.23, No.5 (1980), pp.213-217.

(12) Morrison, W.H., III, Akin, D.E., Ramaswamy, G., and Baldwin, B., Evaluating Chemically Retted Kenaf Using Chemical, Histochemical, and Microspectrophotometric Analyses, Textile Research Journal, Vol.66, No.10 (1996), pp.651-656.

(13) Poscoal Neto, C., Seca, A., Fradinho, D., Coimbra, M.A., Domingues, F., Evtuguin, D., Silvestre, A. and Cavaleiro, J.A.S., Chemical Composition and Structural Features of the Macromolecular Components of Hibiscus Cannabinus Grown in Portugal, Industrial Crops and Products, Vol.5, No.3 (1996), pp.189-196.

(14) Cave, I.D., The Anisotropic Elasticity of the Plant Cell Wall, Wood Science and Technology, Vol.2, No.4 (1968), pp.268-278.

(15) Cousins, W.J., Young's Modulus of Hemicellulose as Related to Moisture Content, Wood Science and Technology, Vol.12, No.3 (1978), pp.161-167.

(16) Cousins, W.J., Elastic Modulus of Lignin as Related to Moisture Content, Wood Science and Technology, Vol.10, No.1 (1976), pp.9-17.

(17) Pipes, R.B. and Pagano, N.J., Interlaminar Stresses in Composite Laminates under Uniform Axial Extension, Journal of Composite Materials, Vol.4, No.4 (1970), pp.538-548. 